\title{
Analysis of the Reactive Components of the Plasma Gap Impedance in Thermionic Energy Converter
}

\author{
Viacheslav P. Zimin, \\ Department of Applied Mathematics \\ Tomsk Polytechnic University (TPU) \\ Lenin Avenue, 30 \\ Tomsk, Russia \\ e-mail: zimin@tpu.ru
}

\author{
Ekaterina A. Isakova \\ Department of Applied Mathematics \\ Tomsk Polytechnic University, TPU \\ Tomsk, Russia \\ Lenin Avenue, 30 \\ e-mail: Ekaterina720@yandex.ru
}

\begin{abstract}
The contribution of the of voltage drop components to the impedance of the plasma gap was analyzed based on the non-stationary models of processes in low temperature cesium plasma. Depending on the phase of the plasma parameters and the properties of the thermionic energy converter the voltage drop components may exhibit both inductive and capacitive properties. Hodographs of the impedance of the plasma arc gap in the converter were calculated and analyzed both for arc and diffusion modes. The effect of temperature resonance on the impedance hodograph of the plasma gap in the arc mode was analyzed. Noted is a significant contribution of the volume ionization thermionic energy converter to the inductive properties of the plasma gap.
\end{abstract}

Keywords - thermionic converter; non-stationary processes; cesium plasma; the impedance of the plasma gap; hodograph; arc mode; the diffusion mode;

\section{INTRODUCTION}

The study of non-stationary processes in the electricity generating channel which is to function is the periodically occurring transient mode is an important step in designing thermionic energy converter [1] working on AC current. It is important study the impedance of both channel designs [2] and the working fluid - the low temperature plasma [3].

Theoretical study of the latter part of the impedance is a difficult task, requiring simulation of the non-stationary processes in low-temperature plasma and dynamic voltampere characteristics of the isothermal thermionic energy converter (TEC). The experimental results show that the impedance of the plasma gap of TEC exhibits both inductive and capacitive properties. Selection of the electrode area and the area occupied by the plasma in the electrode gap is a characteristic of the non-stationary model of plasma gap of TEC [5] used in this paper. The voltage drop across the converter gap operating in direct current mode, is represented as the sum of the voltage drops over the active resistance of each of the areas of the impedance of the plasma gap [3]. For a converter operating in the $\mathrm{AC}$ mode, the voltage drop across these areas can also show capacitive or inductive properties.

The method used in studying the plasma gap involves introduction of small harmonic perturbations into one of the parameters of the TEC. In this paper we vary one of the parameters of the electric circuit - the converter current. The impedance of the plasma gap in TEC forms as a result of a large number of processes in the plasma [3]: surface ionization and volume ionization, collisions of charged particles with neutral ones and with each other, the interaction of the particle flows in the electrode regions, etc. It is important to analyze the contribution of each component of the voltage drop in the reactive properties in bot arc and diffusion modes of the converter.

\section{MODEL AND ANALYSIS OF NON-STATIONARY PROCESSES IN THE PLASMA GAP CONVERTER}

The model of the non-stationary processes in low temperature plasma in arc mode of the thermionic diode and its dynamic volt-ampere characteristics is shown in [5]. Onedimensional model consists of non-stationary equations for plasma density $n(x, t)$

$$
\frac{\partial n}{\partial t}=D_{a} \frac{\partial^{2} n}{\partial x^{2}}+s\left(n, T_{e}\right)
$$

where $D_{a}$ is the ambipolar diffusion coefficient and $s\left(n, T_{e}\right)$ id the stepwise generation of ions in cesium plasma [3];

for the temperature of the electrons $T_{e}(t)$, which is assumed to be constant in space, but changing in time we have

$$
\frac{\partial T_{e}}{\partial t}=F_{T e}\left(\langle n\rangle, T_{e},\left\langle J_{i}\right\rangle,\langle s\rangle, q_{e 0}, q_{e d}, J(t)\right),
$$

where $\left\langle\ldots>\right.$ is average over the gap; $J_{i}-$ ion current density; $q_{e 0}, q_{e d}$ - energy electrons density from the emitter and collector; $J(t)$ - is the current density in the converter;

for an electric circuit we have

$$
\left(F_{E}-F_{C}\right) / e-V_{d}-V_{H}=\left(F_{E}-F_{C}\right) / e-V_{d}-J(t) R_{H} S_{E} \equiv 0,
$$

where $e$-electron charge; $F_{E}, F_{C}$ are the work function of the emitter and collector; $e$ is the electron charge ; $V_{d}$-voltage drop over the plasma gap; $V_{H}$ - load voltage drop; $R_{H}-$ load resistance; $S_{E}$ - electrode area.

The equations for the remaining variables (ion current density, the potential of the space occupied by the plasma) and 
the boundary conditions are taken in a quasi-stationary approximation [5]. This model allows us to study nonstationary processes in the plasma in the frequency $\omega \leq 10^{6} \ldots 10^{8} \mathrm{rad} / \mathrm{s}$.

In [5] we used the boundary conditions, which are characteristic of the arc mode: near-electrode potential barriers decelerate plasma electrons. In [6, 7], we investigated the characteristics of the boundary conditions at the electrodes for monotone potential barriers of different polarity.

For accelerating $\left(V_{E} \leq 0\right)$ near-electrode potential barrier:

$$
\begin{gathered}
J_{e 0}=J_{E e}^{(E)}-\left[J_{r e 0}-A_{E e} J_{e 0}\right] \exp \left(\frac{e V_{E}}{k T_{e 0}}\right), \\
J_{i 0}=J_{E i}^{(E)} \exp \left(\frac{e V_{E}}{k T_{0}}\right)-\left[J_{r i 0}-A_{E i} J_{i 0}\right] \\
\left(1-A_{E e} \exp \left(\frac{e V_{E}}{k T_{e 0}}\right)\right) q_{e 0}= \\
=J_{E e}^{(E)}\left[\frac{2 k T_{E}}{e}-V_{E}\right]-J_{r e 0} \exp \left(\frac{e V_{E}}{k T_{e 0}}\right)\left[\frac{2 k T_{e 0}}{e}-V_{E}\right]
\end{gathered}
$$

for decelerating $\left(V_{E} \geq 0\right)$ near-electrode potential barrier:

$$
\begin{gathered}
J_{e 0}=J_{E e}^{(0)} \exp \left(-\frac{e V_{E}}{k T_{e E}}\right)-\left[J_{r e 0}-A_{E e} J_{e 0}\right], \\
J_{i 0}=J_{E i}^{(0)}-\left[J_{r i 0}-A_{E i} J_{i 0}\right] \exp \left(-\frac{e V_{E}}{k T_{0}}\right), \\
\left(1-A_{E e}\right) q_{e 0}=J_{E e}^{(0)} \exp \left(-\frac{e V_{E}}{k T_{e E}}\right) \frac{2 k T_{E}}{e}-J_{r e 0} \frac{2 k T_{e 0}}{e}, \\
J=J_{e 0}+J_{i 0},
\end{gathered}
$$

where $J_{e 0}, J_{i 0}-$ the electron and ion current density at the emitter; $J_{r e 0}=\gamma_{E e} e n_{0} \bar{v}_{e}\left(T_{e 0}\right), \quad J_{r i 0}=\gamma_{E i} e n_{0} \bar{v}_{i}\left(T_{0}\right) \quad-$ the random current density of electrons and ions from the plasma to the emitter; $\gamma_{E e}, A_{E e}, \gamma_{E i}, A_{E i}$ - proportionality factors for the current chaotic and anisotropy of charged particles and energy flows; $n_{0}-$ the plasma density at the emitter; $\bar{v}_{e}\left(T_{e 0}\right)=\sqrt{\left(8 k T_{e 0}\right) /\left(\pi m_{e}\right)}, \quad \bar{v}_{i}\left(T_{0}\right)=\sqrt{\left(8 k T_{0}\right) /\left(\pi m_{i}\right)}-$ thermal velocities of charged particles; $k-$ is Boltzmann constant; $m_{e}, m_{i}$ - mass of charged particles; $J_{E e}^{(E)}, J_{E i}^{(E)}-$ emission electron and ion currents of the emitter based on the normal Schottky effect [3]; $T_{e 0}, T_{0}$ - temperature of the plasma electrons and ions (atoms) of cesium; $T_{e E}-$ the electron temperature of the emitter; $T_{E}$ - temperature of the emitter.

For decelerating $\left(V_{C} \geq 0\right)$ near-electrode potential barrier:

$$
\begin{gathered}
J_{e d}=\left[J_{r e d}+A_{C e} J_{e d}\right] \exp \left(-\frac{e V_{C}}{k T_{e d}}\right)-J_{C e}^{(E)}, \\
J_{i d}=\left[J_{r i d}+A_{C i} J_{i d}\right]-J_{C i}^{(E)} \exp \left(-\frac{e V_{C}}{k T_{d}}\right), \\
\left(1-A_{C e} \exp \left(-\frac{e V_{C}}{k T_{e d}}\right)\right) q_{e d}= \\
=J_{r e d} \exp \left(-\frac{e V_{C}}{k T_{e d}}\right)\left[\frac{2 k T_{e d}}{e}+V_{C}\right]-J_{C e}^{(E)}\left[\frac{2 k T_{C}}{e}+V_{C}\right],
\end{gathered}
$$

for accelerating $\left(V_{C} \leq 0\right)$ near-electrode potential barrier:

$$
\begin{gathered}
J_{e d}=\left[J_{r e d}+A_{C e} J_{e d}\right]-J_{C e}^{(0)} \exp \left(\frac{e V_{C}}{k T_{e C}}\right), \\
J_{i d}=\left[J_{r i d}+A_{C i} J_{i d}\right] \exp \left(\frac{e V_{C}}{k T_{d}}\right)-J_{C i}^{(0)}, \\
\left(1-A_{C e}\right) q_{e d}=J_{r e d} \frac{2 k T_{e d}}{e}-J_{C e}^{(0)} \exp \left(\frac{e V_{C}}{k T_{e C}}\right) \frac{2 k T_{C}}{e}, \\
J=J_{e d}+J_{i d},
\end{gathered}
$$

where $J_{e d}, J_{i d}$ - the electron and ion current density at the collector; $J_{\text {red }}=\gamma_{C e} e n_{d} \bar{v}_{e}\left(T_{e d}\right), \quad J_{\text {rid }}=\gamma_{C i} e n_{d} \bar{v}_{i}\left(T_{d}\right)-$ the random current density of electrons and ions from the plasma to the collector; $\gamma_{C e}, A_{C e}, \gamma_{C i}, A_{C i}$ - proportionality factors for the current chaotic and anisotropy of charged particles and energy flows; $n_{d}-$ the plasma density at the collector; $\bar{v}_{e}\left(T_{e d}\right)=\sqrt{\left(8 k T_{e d}\right) /\left(\pi m_{e}\right)}, \quad \bar{v}_{i}\left(T_{d}\right)=\sqrt{\left(8 k T_{d}\right) /\left(\pi m_{i}\right)}-$ thermal velocities of charged particles; $J_{C e}^{(E)}, J_{C i}^{(E)}$ - emission electron and ion currents of the collector based on the normal Schottky effect; $T_{e d}, T_{d}$ - temperature of the plasma electrons and ions (atoms) of cesium; $T_{e C}-$ the electron temperature of the collector; $T_{C}-$ temperature of the collector.

Research results monotone boundary conditions allowed us to apply the model [5] for the analysis of non-stationary processes in the diffusion mode of TIC operation.

Experiments [4] show that TEC impedance exhibits both inductive and capacitive properties. Selection of the electrode area and the area occupied by the plasma in the electrode gap is a characteristic of the non-stationary model we use. For direct current each of these areas can be represented as an equivalent resistance. These areas will show some reactive properties for alternating current. It is therefore of interest to investigate with the help of the model (1) - (3) the contribution of the processes that lead to the impedance of each region in the reactive properties of the plasma impedance gap in the converter. 
If we write perturbations of the current, and the voltage as as $J(t)=J_{0}+\delta J \cdot \sin \left(\omega t+\varphi_{J}\right)$ and $V_{H}(t)=V_{H 0}+\delta V_{H} \cdot \sin \left(\omega t+\varphi_{V H}\right)$, relative to the values at the point of stationary volt-ampere characteristics $\left(V_{H 0}, J_{0}\right)$, then at small amplitudes $\delta J / J_{0}<<1$ and $\delta V_{H} / V_{H 0}<<1$, the impedance of the plasma gap in TEC can be written as

$$
z=\frac{\dot{V}_{H}}{\dot{J}}=\frac{\delta V_{H}}{\delta J} e^{i\left(\varphi_{V H}-\varphi_{J}+\pi\right)} .
$$

In our investigation, we are given $\varphi_{J}=0$ and it is sufficient to know $\varphi_{V H}$ to study the reactive properties of $z$. At $0<\varphi_{V H}<\pi$ the impedance has capacitive properties, and at $\pi<\varphi_{V H}<2 \pi$ it has inductive properties. Perturbation $\tilde{V}_{H}(t)$ is determined by the following expressions [5]

$$
\tilde{V}_{H}=\left(\tilde{F}_{E}-\tilde{F}_{C}\right) / e-\tilde{V}_{d}, \tilde{V}_{d}=-\tilde{V}_{E}+\tilde{V}_{N}+\tilde{V}_{K}+\tilde{V}_{L}-\tilde{V}_{C},
$$

where $\tilde{F}_{E}, \tilde{F}_{C}, \tilde{V}_{d}$ are the perturbations of the work function of output of the emitter and collector and of the voltage drop across the electrode gap transmitter; $\tilde{V}_{E}, \tilde{V}_{N}, \tilde{V}_{K}, \tilde{V}_{L}, \tilde{V}_{C}$ are the perturbations of the voltage drop components: in a sheet at the emitter, due to the collision of charged and neutral particles in the plasma, due to Coulomb collisions, due to the diffusion components in the sheet at the collector. Then based on (4) and (5) and given $\varphi_{J}=0$ we can write

$$
z=z_{F E}-z_{F C}+z_{E}-z_{N}-z_{K}-z_{L}+z_{C}
$$

Thus, the impedance of the plasma gap in TEC is equal to the algebraic sum of the impedances, each of which is formed, just as a corresponding voltage drop, due to certain physical processes in the plasma around and on electrodes of the converter: $z_{E}, z_{C}-$ non-equilibrium processes in the regions near the electrode; $z_{N}-$ scattering of charged particles on neutrals; $z_{K}-$ Coulomb collisions; $z_{L}$ - differences in plasma concentrations near electrodes; $z_{F E}, z_{F C}$ - the influence of plasma parameters on the $F_{E}, F_{C}$ values, e.g., due to the Schottky effect.

We examine the nature of reactivity of each $l$-th term in (6) ( $l=F E, F C, E, N, K, L, C$ ) based on the behavior of $\varphi_{V l}$, because the relation $\varphi_{z l}=\varphi_{V l}+\pi$ is valid in this case as well. In turn the values of $\varphi_{V l}$ depend on the parameters of the nonstationary plasma $\tilde{n}(\xi, t), \tilde{T}_{e}(t)$ and current $\tilde{J}(t)$.

Previous studies show [5], that perturbations $F_{E}, F_{C}$ due to Schottky effect are negligible, i.e. $\tilde{F}_{E}(t) \approx 0, \tilde{F}_{C}(t) \approx 0$, and main contribution to impedance $z$ comes from the impedances of the regions near the electrode $z_{E}, z_{C}$ and impedance of the region containing plasma $z_{p}=z_{N}+z_{K}+z_{L}$.
We perform the following analysis to study the phase of the $l$-th component of the voltage drop. Linearizing $\tilde{V}_{l}(t)$ with respect to $\tilde{n}(\xi, t), \tilde{T}_{e}(t), \tilde{J}(t)$, we obtain

$$
\tilde{V}_{l}(t)=a_{l T}^{j} \tilde{T}_{e}(t)+a_{l n}^{j} \tilde{n}(t)+a_{l J}^{j} \tilde{J}(t)
$$

where $a_{l(T, n, J)}^{j}$ the first derivatives of $\tilde{V}_{l}(t)$ with respect to: electron temperature, plasma density and current at the stationary point. Upper index $j$ is introduced only for $l=E, C$ and denotes the polarity of the voltage drop near electrodes: $j=1$ for $V_{E}<0, \quad V_{C}<0$ and $j=0$ for $V_{E}>0, V_{C}>0$. For $l=N$ $\tilde{n}(t)=\langle\tilde{n}(x, t)\rangle_{x}$, i.e. the non-stationary plasma density is averaged over $x \in[0, d]$.

Using the results of the study of the temperature resonance from [8], we estimate the domain of $\varphi_{T}$ and $\varphi_{n}$ in the frequency range $[0, \infty)$. For $\varphi_{T}$, according to the estimates from [8], we have

$$
-\pi / 2 \leq \varphi_{T} \leq 0,0 \leq \varphi_{T} \leq \pi / 2
$$

and for $\varphi_{n}$ we have

$$
0 \leq \varphi_{n}<\pi / 2,-\pi \leq \varphi_{n}<0
$$

\section{SIMULATION RESULTS AND DISCUSSION}

Based on studies in the sign of the coefficients $a_{l(T, n, J)}^{j}$ in (7), the use of inequalities (8), (9) and the expression (5), (6) we obtain estimates of possible reactive properties of each component $z_{l}$ in relation to the impedance of the plasma clearance $z$. For example, when component $z_{E}$ at $0 \leq \varphi_{T} \leq \pi / 2$ and $0 \leq \varphi_{n}<\pi / 2$ can only exibit inductive properties. Results show that depending on the values of plasma parameters at the stationary point in volt-ampere characteristics any term in (6) in principle may exhibit both inductive and capacitive properties.

Fig. 1 shows the hodographs of the impedance of the plasma gap, whose coordinates Rez and Imz are computed from (4) for different points of stationary volt-ampere characteristics, computed for the following TEC parameters: $T_{E}=1800 \mathrm{~K}, T_{C}=950 \mathrm{~K}, p_{\mathrm{Cs}}=4$ Torr, $d=0.025 \mathrm{~cm}, J_{E e}=28.9$ $\mathrm{A} / \mathrm{cm}^{2}$. For the arc mode we set $\delta J / J_{0}=0.1$, and for diffusion mode we set $\delta J / J_{0}=0.005$.

The typical hodographs for two current densities on the arc branch of the volt-ampere characteristics of TEC $\left(J_{0}, \mathrm{~A} / \mathrm{cm}^{2}\right.$ : $1-1.5 ; 2-1.0)$ are shown in panel (a). Panel (b) shows the hodographs at $J_{0}=0.71 \mathrm{~A} / \mathrm{cm}^{2}$ on the diffusion branch of voltampere characteristics of the converter with $(\bullet)$ and without (x) accounting for ionization in plasma. The numbers at the $x$ 's and dots on the hodographs show the frequencies of the perturbations of current density times $10^{-3}$. 
(a)

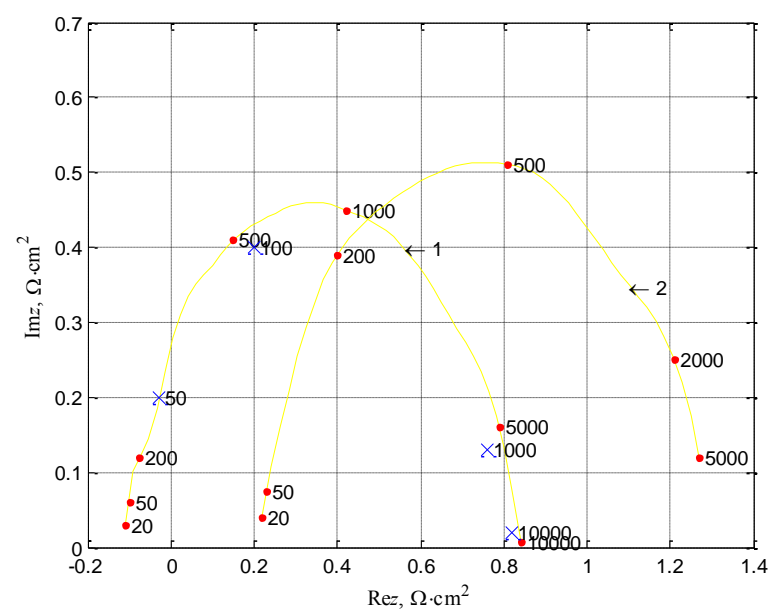

(b)

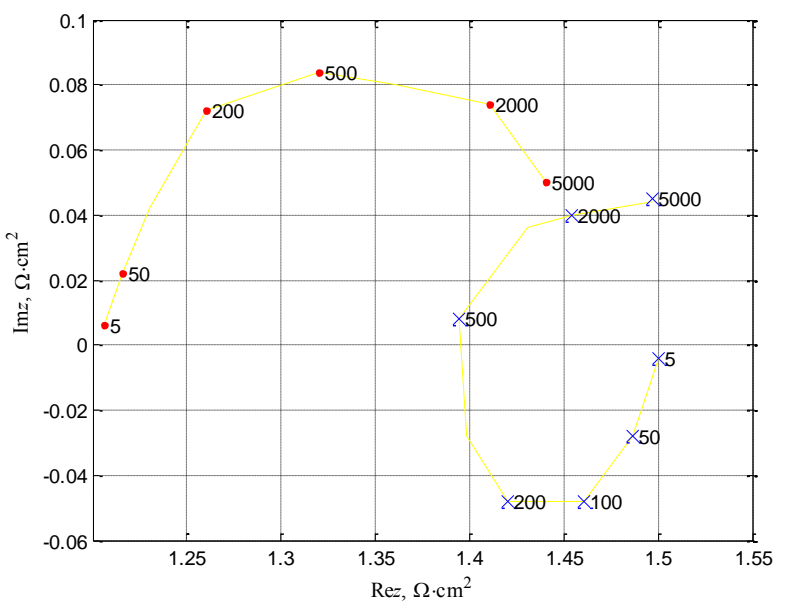

Fig. 1. Hodographs of the impedance of the plasma gap of the converter: (a) - arc mode; (b) - diffusion mode

The impedance of the plasma gap in the arc mode shows only the inductive properties (Imz $>0$ ). The reactive component has a maximum in the area of transition from the diffusion mode to the arc mode. Moving the operating point to higher current densities leads to the decrease in the maximum value of Imz. For example, for $J_{0}=25.5 \mathrm{~A} / \mathrm{cm}^{2}$ the maximum $\operatorname{Im} z \approx 0.008 \Omega \cdot \mathrm{cm}^{2}$ at $\omega=5 \cdot 10^{5} \mathrm{rad} / \mathrm{s}$. Such a marked decrease in the reactive properties of the impedance of the plasma gap is due to a significant increase in the plasma density in the electrode gap.

The x's on panel (a) curve 1 show the hodograph of the impedance of the plasma gap, computed ignoring the electron temperature resonance leading to noticeable increase of nonstationary amplitude of electrons $\delta T_{e} / T_{e 0}=\delta T_{e} / T_{e 0}(\omega)$ when the frequency of perturbation increases [8]. For this purpose, the amplitude $\delta \bar{T}_{e}\left(\omega^{*}\right)=$ const was fixed to the amplitude of perturbations, computed for low frequencies $\omega^{*}$, when the temperature response is not yet manifested. From a comparison of the values of the impedance of the plasma gap obtained with $(\bullet)$ and without $(\mathrm{x})$ taking into the account the temperature of the resonance, we see little change in the differential resistance of the stabilized dynamic volt-ampere characteristics calculated with and without taking into account the temperature of the resonance. The hysteresis characteristics of the properties remain virtually identical. But the maximum value of hysteresis and stabilization of dynamic volt-ampere characteristics appears at much lower frequencies $\omega$ for points of hodograph of the impedance of the plasma gap, calculated without taking into account the temperature resonance.

The impedance of the plasma gap in diffusion mode of TEC exhibits both inductive and capacitive properties (Figure 1 , panel b). While capacitive properties are manifested only without taking into account the volume ionization (Figure 1, (b) $\mathrm{x}$ ) for the current densities are close to the impedance of the plasma gap quasi-saturation current, at lower currents $J_{0} \leq 0,45 \mathrm{~A} / \mathrm{cm}^{2}$ the capacitive properties are also manifested even with taking into account the volume ionization $(\bullet)$. The hodographs of the impedance of the plasma gap calculated in such approximations differ little from each other.

\section{CONCLUSION}

We have shown that the behavior of the plasma gap impedance in the converter operating in different modes of operation is complicated. Depending on the parameters of thermionic energy converter and the state of the plasma at the operating point on the volt-ampere characteristics components of the plasma clearance impedance $c$ can exhibit both inductive and capacitive properties. The effect of the temperature resonance plays a significant role in the arc mode of the converter.

With the reduction of the effect of volume ionization on the processes in the electrode gap, manifestation of capacitive properties of the impedance in TEC increases. This may explain the appearance of intermediate frequency stabilization [4] in the field of discharge pinching on the experimentally obtained volt-ampere characteristics, where regions of plasma parameters characteristic of the arc and diffusion modes of TEC coexist.

\section{ACKNOWLEDGEMENTS}

Author thanks Aleksey V. Zimin for the English translation and editing of the manuscript.

\section{REFERENCES}

[1] V.V. Sinyavsky "Methods and means of experimental research and test reactor thermionic assemblies," Moscow: Energoatomisdat, 2000, p. 375.

[2] M.A. Mendelbaum, V.D. Eskov "Thermionic converter in load switching mode," Proceedings of the Academy of Sciences of the USSR. Energy and Transport 1983, number 6, pp. 147-151. 
[3] F.G. Baksht, G.A. Dyuzhev, A.M. Marcinowski, B.J. Mojzes, G.E. Picos, E.B. Sonin, V.G. Yuryev "Thermionic converter and low temperature plasma," Moscow: Nauka, 1973, p. 480.

[4] N.S. Lidorenko, A.I. Loshkarev, V.D. Bondarenko "The dynamic characteristics of plasma diode in the low-voltage arc discharge mode. II. Experimental study of dynamic characteristics, " Technical Physics, 1973, Volume 43, pp. 1203-1211.

[5] A.L. Deynezhenko, V.P. Zimin "Numerical simulation of transient voltampere characteristics of the diode in the plasma arc mode," Proceedings of the Academy of Sciences of the USSR. Technical sciences, 1987, № 6, pp. 84-87.

[6] V.P. Zimin "Research of features of the plasma boundary conditions at the thermionic emitter diode," Bulletin of the Tomsk Polytechnic University, 2013, Volume 322, number 2, pp. 11-15.

[7] V.P. Zimin "Research of features of the plasma boundary conditions at the collector thermionic diode," Bulletin of the Tomsk Polytechnic University, 2013, Volume 323, number 2, pp. 158-163.

[8] V.P. Zimin "Investigation of resonance in the low temperature nonstationary plasma" [Proceedings of VIII-th All-Union Conference on Low Temperature Plasma Physics, Minsk, 1991, pp. 27-28]. 